SECTION 4. Computer science, computer engineering and automation.

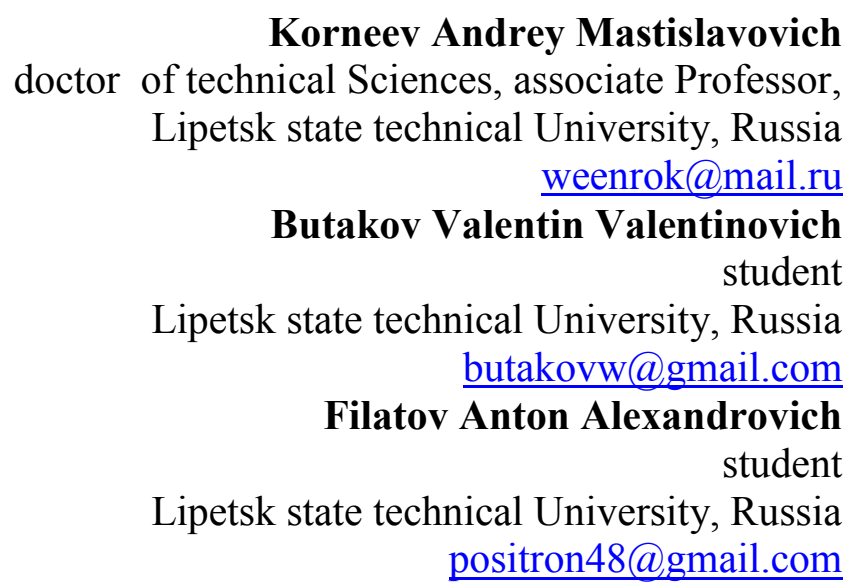

\title{
DISCRETE-VARIABLE MODELING OF TECHNOLOGICAL PROCESS
}

Abstract: Presents a methodology that allows to present technological process in the form of cells, each of which corresponds to one technological unit or redistribution. Detailing the form of inputs, States, and the outputs specifications of raw materials, technological parameters and properties of the finished products, you can simulate the processing technology of all types of products. The use of machines and iterative chains will describe any complex process and manage technology.

Key words: discrete cellular-hierarchical systems, automatic, iterative chains, structural modeling, technology, alphabet machine, atomicity alphabet, Jegalkin polynomial.

\section{УДК 62.529}

\section{ДИСКРЕТНО-АГРУМЕНТНОЕ МОДЕЛИРОВАНИЕ ТЕХНОЛОГИЧЕСКОГО ПРОЦЕССА}

\begin{abstract}
Аннотация: Представлена методика, позволяющая представить технологический прочесс в виде клеток, каждая из которых соответствует отдельному технологическому агрегату или переделу. Описав в виде входов, состояний и выходов характеристики сырья, технологических параметров и свойств готовой продукиии, можно моделировать технологии обработки любых видов продукиии. Использование автоматов, итеративных иепей позволит описать любой сложный технологический прочесс и осуществлять управление технологией.

Ключевые слова: дискретные клеточно-иерархические системы, автомат, итеративные цепи, структурное моделирование, технология, алфавит автомата, значность алфавита, полином Жегалкина.
\end{abstract}

Любой сложный технологический процесс состоит технологических стадий обработки продукции. Каждую из технологических операций можно представить в виде клеток, описанных в виде входов и выходов (параметры сырья, технологические факторы, свойства готовой продукции). Тогда модель технологического процесса можно представить в виде итеративной цепи $[1 ; 2]$.

Итеративная цепь - это композиция неоднородных клеток (автоматов) различной степени сложности, имеющих последовательное соединение, в которых внутренние выходы предыдущих клеток соединяются с внутренними входами последующих клеток. Дискретная клеточно-иерархическая система - это система 
многоуровневой структуры, представляющая собой композицию сложных автоматов, которые разбиваются на отдельные элементарные автоматы (клетки), формирующие итеративные цепи [3].

Автомат, который описывает дискретную клеточно-иерархическую систему, моделируется внутренними цепями клеток, причем каждая такая клетка из внутренней цепи моделирует некоторую стадию обработки дискретной клеточно-иерархической системы. Любой автомат в цепи $i$-того уровня $S(i)$ дискретной клеточноиерархической системы может быть представлен в виде итеративной цепи $i+1$-го уровня, при этом входы итеративной цепи является входами данного автомата, а выходы - выходами данного автомата [4]. На рис.1 представлен синтез внутренних итеративных цепей.

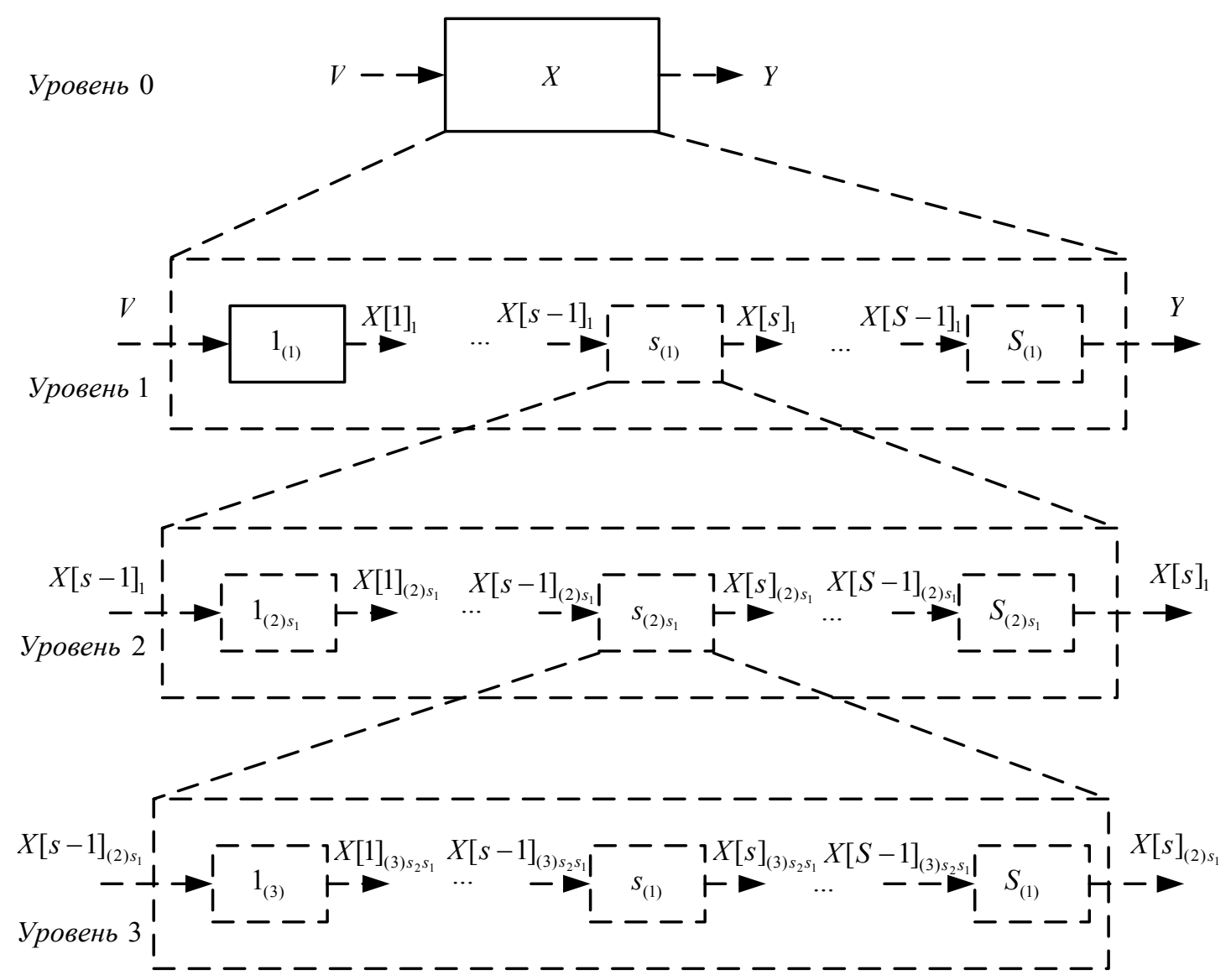

Рисунок 1 - Дискретная клеточно-иерархическая система

На вход в первую клетку внутренней цепи подаются данные о факторах, реализованных на предшествующей стадии обработки (предыдущей клетки внешней цепи). На вход в последующие клетки внутренней цепи подаются факторы, реализованных в предыдущей клетки внутренней цепи.

Для каждой клетки, описывающей технологическую операцию на некотором агрегате, определяется свое уравнение перехода:

$$
\left[\begin{array}{c}
x_{s_{k} 1} \\
\ldots \\
x_{s_{k} m_{\mathrm{s}}}
\end{array}\right]=f\left[\begin{array}{c}
x_{(s-1)_{k} 1} \\
\ldots \\
x_{(s-1)_{k} m_{\mathrm{s}-1}}
\end{array}\right],
$$

где $m_{s}$ - количество внутренних выходов $s_{k}$-й клетки . 
Если имеется $\mathrm{N}$ технологических факторов, а каждый фактор разбивается на $\mathrm{K}$ интервалов, то будет получено $L=K^{N}$ различных комбинаций всего комплекса технологических факторов. Все комбинации интервалов технологических факторов можно представить в виде кортежей вида: $\left(k_{1}, \ldots, k_{n}\right), k_{i} \in K_{i}$, где $k_{i}$ - номер интервала iтого технологического фактора, $K_{i}$ - множество интервалов, на которые разбит диапазон изменения і-того технологического фактора [5; 6].

Диапазон имения выходного свойства разбивается три (в общем случае) интервала: интервал, регламентированный ГОСТ; интервал со значениями ниже ГОСТ; интервал со значениями выше ГОСТ.

Для каждого технологического подпространства определяется количество $n_{i}$ попадания опытов $v_{i}$ в данное технологическое подпространство, а также частоту попадпний выходных свойств $y$ в каждый интервал $y_{i}$ диапазона изменения выходного свойства (показателя качества). Результаты описания технологических подпространств показаны в табл. 1.

Таблица 1

Описание технологических подпространств

\begin{tabular}{|c|c|c|c|c|c|c|}
\hline \multirow{2}{*}{$\begin{array}{l}\text { Технологическое } \\
\text { подпространство, } \\
T_{i}\end{array}$} & \multirow{2}{*}{$\begin{array}{l}\text { Кортеж } \\
\text { интервалов } \\
\text { технологических } \\
\text { факторов }\end{array}$} & \multirow{2}{*}{$\begin{array}{l}\text { количество } \\
\text { попадания } \\
\text { опытов, } \\
n_{i} \\
\end{array}$} & \multicolumn{4}{|c|}{$\begin{array}{l}\text { частота попадания } \\
\text { интервалы выхода, } y\end{array}$} \\
\hline & & & $y_{1}$ & $y_{2}$ & $\ldots$ & $y_{m}$ \\
\hline$T_{1}$ & $\left(k_{11}, \ldots, k_{1 n}\right)$ & $n_{1}$ & $n_{11}$ & $n_{12}$ & $\ldots$ & $n_{1 m}$ \\
\hline$T_{2}$ & $\left(k_{21}, \ldots, k_{2 n}\right)$ & $n_{2}$ & $n_{21}$ & $n_{21}$ & $\cdots$ & $n_{2 m}$ \\
\hline$\ldots$ & $\ldots$ & $\ldots$ & $\ldots$ & $\ldots$ & $\ldots$ & $\ldots$ \\
\hline$T_{L}$ & $\left(k_{L 1}, \ldots, k_{L n}\right)$ & $n_{L}$ & $n_{L 1}$ & $n_{L 2}$ & $\cdots$ & $n_{L m}$ \\
\hline
\end{tabular}

Закодируем все технологические пространства и все интервалы выходного свойства (показателя качества) своими кодами в порядке возрастания двоичного кода. Размер двоичного кода: $B=\min \left\{n \mid L \leq 2^{n}\right\}$, где $L$ - количество технологических подпространств. Результаты кодирования представлены в табл.2.

Таблица 2

Кодирование технологических подпространств

\begin{tabular}{|l|l|l|l|l|}
\hline $\begin{array}{l}\text { Технологическое } \\
\text { подпространство, } \\
T_{i}\end{array}$ & $\begin{array}{l}\text { Кортеж } \\
\text { интервалов } \\
\text { технологических } \\
\text { факторов }\end{array}$ & $\begin{array}{l}\text { Код } \\
\text { технологического } \\
\text { подпространства, } \\
B\end{array}$ & $\begin{array}{l}\text { Интервал } \\
\text { выхода, y }\end{array}$ & $\begin{array}{l}\text { Код } \\
\text { интервала } \\
\text { выхода, y }\end{array}$ \\
\hline$T_{1}$ & $\left(k_{11}, \ldots, k_{1 n}\right)$ & 0000 & $y_{1}^{\prime}$ & 10 \\
\hline$T_{2}$ & $\left(k_{21}, \ldots, k_{2 n}\right)$ & 0001 & $y_{2}^{\prime}$ & 11 \\
\hline$\ldots$ & $\ldots$ & $\ldots$ & $\ldots$ & $\ldots$ \\
\hline$T_{L}$ & $\left(k_{L 1}, \ldots, k_{L n}\right)$ & 1100 & $y_{L}^{\prime}$ & 01 \\
\hline
\end{tabular}

Состояния и выходы получившегося автомата удобно описать с помощью полинома Жегалкина: 


$$
P\left(X_{1}, \ldots, X_{2}\right)=a \oplus a_{1} X_{1} \oplus a_{2} X_{2} \oplus \ldots \oplus a_{B} X_{B} \oplus a_{12} X_{1} X_{2} \oplus a_{13} X_{1} X_{3} \oplus \ldots \oplus a_{1 . . B} X_{1} \ldots X_{B}
$$

Полиномы Жегалкина составляются для каждого двоичного бита кода выхода автомата. Эти полиномы содержат зависимости двоичного бита кода выхода автомата от кода текущего состояний автомата (текущее технологическое подпространство).

Построим модель этапа технологического процесса. Имеются статистические данные о данном этапе. Исследуются 3 технологических фактора, а диапазон изменения каждого фактора от минимального до максимального значения разбивается на 3 интервала. Также, диапазон изменения контролируемого выходного свойства от минимального до максимального значения разбивается на 2 интервала. Первый интервал - интервал, предусмотренный ГОСТ. Второй интервал - значения выходного свойства, не предусмотренные ГОСТ.

Закодируем все технологические пространства в двоичном коде [9; 10]. Размер двоичного кода, которым кодируются технологические подпространства определяется по формуле: $B=\min \left\{n \mid 27 \leq 2^{n}\right\}=1$. Пример кодирования представлен в табл.3.

Таблица 3

\section{Пример кодирования технологических подпространств}

\begin{tabular}{|c|c|c|c|c|c|c|}
\hline \multicolumn{3}{|c|}{$\begin{array}{c}\text { Номера интервалов } \\
\text { технологических факторов }\end{array}$} & \multirow{2}{*}{$\begin{array}{c}\text { Код } \\
\text { технол. } \\
\text { подпрос- } \\
\text { транства }\end{array}$} & \multicolumn{2}{|c|}{$\begin{array}{l}\text { Частота попадания в } \\
\text { интервалы качества }\end{array}$} & \multirow{2}{*}{$\begin{array}{c}\text { Код } \\
\text { интервала } \\
\text { качества }\end{array}$} \\
\hline Фактор 1 & Фактор 2 & Фактор 3 & & Интервал 0 & Интервал 1 & \\
\hline 1 & 1 & 1 & 00000 & 0 & 1 & 1 \\
\hline 1 & 2 & 1 & 00001 & 0,6 & 0,4 & 0 \\
\hline 2 & 2 & 1 & 00010 & 0,44 & 0,56 & 1 \\
\hline 3 & 2 & 1 & 00011 & 0,69 & 0,31 & 0 \\
\hline 1 & 3 & 1 & 00100 & 1 & 0 & 0 \\
\hline 2 & 3 & 1 & 00101 & 0,7 & 0,3 & 0 \\
\hline 3 & 3 & 1 & 00110 & 0,46 & 0,54 & 1 \\
\hline 2 & 1 & 2 & 00111 & 0 & 1 & 1 \\
\hline 1 & 2 & 2 & 01000 & 0,5 & 0,5 & 0 \\
\hline 2 & 2 & 2 & 01001 & 0,65 & 0,35 & 0 \\
\hline 3 & 2 & 2 & 01010 & 0,54 & 0,46 & 0 \\
\hline 1 & 3 & 2 & 01011 & 0,57 & 0,43 & 0 \\
\hline 2 & 3 & 2 & 01100 & 0,57 & 0,42 & 0 \\
\hline 3 & 3 & 2 & 01101 & 0,54 & 0,46 & 0 \\
\hline 2 & 2 & 3 & 01110 & 0,29 & 0,71 & 1 \\
\hline 3 & 2 & 3 & 01111 & 0,67 & 0,33 & 0 \\
\hline 2 & 3 & 3 & 10000 & 0,7 & 0,3 & 0 \\
\hline 3 & 3 & 3 & 10001 & 0,43 & 0,57 & 1 \\
\hline
\end{tabular}

Полином Жегалкина для кода интервала выходного свойства от битов кода технологического подпространства имеет вид:

$P\left(X_{1}, X_{2}, X_{3}, X_{4}, X_{5}\right)=a_{00000} \oplus a_{00001} X_{1} \oplus a_{00010} X_{2} \oplus a_{00011} X_{1} X_{2} \oplus a_{00100} X_{3} \oplus a_{00101} X_{1} X_{3} \oplus$

$\oplus a_{00110} X_{2} X_{3} \oplus a_{00111} X_{1} X_{2} X_{3} \oplus a_{01000} X_{4} \oplus a_{01001} X_{1} X_{4} \oplus a_{01010} X_{2} X_{4} \oplus a_{01011} X_{1} X_{2} X_{4} \oplus$

$\oplus a_{01100} X_{3} X_{4} \oplus a_{01101} X_{1} X_{3} X_{4} \oplus a_{01110} X_{2} X_{3} X_{4} \oplus a_{01111} X_{1} X_{2} X_{3} X_{4} \oplus a_{10000} X_{5} \oplus a_{10001} X_{1} X_{5} \oplus$

$\oplus a_{10010} X_{2} X_{5} \oplus a_{10011} X_{1} X_{2} X_{5} \oplus a_{10100} X_{3} X_{5} \oplus a_{10101} X_{1} X_{3} X_{5} \oplus a_{10110} X_{2} X_{3} X_{5} \oplus a_{10111} X_{1} X_{2} X_{3} X_{5} \oplus$

$\oplus a_{11000} X_{4} X_{5} \oplus a_{11001} X_{1} X_{4} X_{5} \oplus a_{11010} X_{2} X_{4} X_{5} \oplus a_{11011} X_{1} X_{2} X_{4} X_{5} \oplus a_{11100} X_{3} X_{4} X_{5} \oplus$

$\oplus a_{11101} X_{1} X_{3} X_{4} X_{5} \oplus a_{11110} X_{2} X_{3} X_{4} X_{5} \oplus a_{11111} X_{1} X_{2} X_{3} X_{4} X_{5}$. 
Методом треугольника найдем коэффициенты полинома Жегалкина:

$$
\begin{array}{lllllllll}
a_{00000}=1 & a_{00001}=1 & a_{00010}=0 & a_{00011}=0 & a_{00100}=1 & a_{00101}=1 & a_{00110}=1 & a_{00111}=0 & a_{01000}=1 \\
a_{01001}=1 & a_{01010}=0 & a_{01011}=0 & a_{01100}=1 & a_{01101}=1 & a_{01110}=0 & a_{01111}=1 & a_{10000}=1 & a_{10001}=0
\end{array}
$$

Остальные коэффициенты равны 0, так как не все входные комбинации полинома Жегалкина используются и значения этих коэффициентов для моделирования не играют роли.

Значит, полином Жегалкина, описывающий зависимость бита кода интервала выходного свойства от битов кода технологического подпространства имеет вид: $P\left(X_{1}, X_{2}, X_{3}, X_{4}, X_{5}\right)=1 \oplus X_{1} \oplus X_{3} \oplus X_{1} X_{3} \oplus X_{2} X_{3} \oplus X_{4} \oplus X_{1} X_{4} \oplus X_{3} X_{4} \oplus X_{1} X_{3} X_{4} \oplus$ $\oplus X_{1} X_{2} X_{3} X_{4} \oplus X_{5}$ где $X_{1}, \ldots, X_{2}$ - биты кода технологического подпространства.

Таким образом, имеем модель этапа технологического процесса в виде автомата. Каждому коду технологического подпространства ставится в однозначное соответствие символ алфавита состояния автомата. Аналогично, каждому коду выходного свойства (критерия качества) ставится в однозначное соответствие символ алфавита выхода автомата. Таблица переходов для автомата задается в виде полинома Жегалкина.

\section{Список литературы}

1. Корнеев, А.М. Дискретное моделирование систем автоматизации и управления [Текст]: Монография / С.Л. Блюмин, А.М. Корнеев. - Липецк: ЛЭГИ, 2005. - 132 с.

2. Корнеев, А.М. Использование итеративных цепей для описания многостадийных пространственно-распределенных производственных систем [Текст]/ А.М. Корнеев, В.Н. Малыш, Т.А. Сметанникова // Вестник Российского университета дружбы народов. Серия: Инженерные исследования, 2012. - №2. - С. 78-84.

3. Корнеев А.М. Структурное моделирование и поисковая оптимизация дискретных клеточно-иерархических систем. Автореферат диссертации на соискание ученой степени доктора технических наук.

4. Корнеев, А.М. Методы идентификации сквозной технологии производства металлопродукции [Текст]: Монография / А.М. Корнеев. - Липецк: ЛГПУ, 2009. $286 \mathrm{c.}$

5. Захаров Н. Г., Рогов В. Н. Синтез цифровых автоматов: Учебное пособие / Н. Г. Захаров, В. Н. Рогов. - Ульяновск: УлГТУ, 2003.

6. Лазарев В. Г., Пийль Е. И. Синтез управляющих автоматов. - 3-е изд., перераб. и доп.-М.: Энергоатомиздат, 1989.- 328c.

7. Максимей, И.В., Левчук, В.Д., Маслович, С.Ф. Инструментарий имитационного моделирования дискретных вероятностных технологических процессов производства // Проблемы программирования. 2003. №3. С92-99.

8. Штерензон, В.А. Моделирование технологических процессов. Конспект лекций. Екатеринбург: Изд-во РГППУ, 2010. - 66с.

9. Муромцев, Ю.Л. Моделирование и оптимизация сложных систем при изменениях состояния функционирования / Ю.Л. Муромцев, Л.Н. Ляпин, О.В. Попова. Воронеж : Изд-во ВГУ, 1992. - 164с.

10. Бусленко, Н.П. Моделирование сложных систем / Н.П. Бусленко. - М.: Наука, 1988. $-356 \mathrm{c}$. 